\title{
Dynamic noise mapping: a map-based interpolation between noise measurements with high temporal resolution
}

\author{
Weigang $W^{1}{ }^{1}$, Timothy Van Renterghem ${ }^{1}$, Bert De Coensel ${ }^{1}$, and Dick Botteldooren ${ }^{1}$ \\ ${ }^{1}$ Email: timothy.van.renterghem@intec.ugent.be; Tel:+32 9264 3634; Fax: +32 92649969 ; \\ Department of Information Technology, Ghent University, Sint-pietersnieuwstraat 41, 9000 \\ Ghent, Belgium
}

\begin{abstract}
Since the introduction of the Environmental Noise Directive, strategic noise mapping has been used as a tool for noise policy in many European countries. Although these strategic noise maps have their merits, they also have some shortcomings: accuracy in predicted noise levels in shielded or quiet areas is not very high, the maps fail to capture sounds that are less easy to predict, and above all the dynamics of the sound environment is not included. However, these dynamics might be important to evaluate sleep disturbance and noise annoyance. In this paper, a model to dynamically (every 15 minutes) update a noise map based on measurements is proposed. This model relies on reasonable good source and propagation models and a not-very-dense measurement network. The least mean squares method (LMS) is used for tuning model parameters. To avoid an under-determined system, the number of degrees of freedom is reduced by grouping the sources and propagation paths into different categories. Source strengths and propagation path attenuations in the same category are corrected by offsetting the same small values from their base levels. The map-based interpolation is performed jointly on $L_{A e q}, L_{10}$ and $L_{90}$, and takes into account 1/3octave band spectra. The efficiency of the proposed method was validated in a case study in the Katendrecht district of Rotterdam, the Netherlands. The results showed that more than $75 \%$ of the $L_{A e q}$ predictions are closer to the measurement than the ab initio calculations based on traffic data. Values for $L_{10}$ and $L_{90}$ are closer to measurements for $55 \%$ and $90 \%$ of the observations, respectively.
\end{abstract}

keywords: dynamic noise map; interpolation; noise measurement; GIS; LMS algorithm

\section{Introduction}

Strategic noise maps have been calculated in many European countries as obliged by the Environmental Noise Directive issued in 2002. Since then, such maps have become an important noise policy instrument, and allow to identify black spots and to draw statistics of population exposure to environmental noise $[1,2,3,4,5]$. Case studies and in-situ measurements $[6,7,8]$ show that discrepancies between calculated and measured acoustical indicators can become quite large, especially in highly shielded urban zones. This inaccuracy mainly stems from assumptions made by the 
source or propagation models commonly applied. Simplifications implemented to allow fast evaluation could be a reason as well. Among others, the influence of meteorological effects, intermediate city canyons [9, 10], green roofs [11], roof details [12] and terrain details $[13,14]$ is not sufficiently captured.

Strategic noise maps, by concept, do not attempt to cover all environmental sound sources. For example, traffic on minor roads is seldom considered due to lack of base data. Other sources such as recreation (pubs, music events,...), (un)loading near shops, construction noise etc. are very difficult to grasp in strategic maps due to their unpredictable and episodic nature. However, these might have a strong impact on the urban soundscape.

Furthermore, the temporal variation is neglected in strategic noise maps, aiming at providing yearly averaged noise indicators. This might conflict with the more temporary perception of environmental noise by city dwellers. In addition, in order to estimate health-related impacts resulting from noise exposure, more detailed temporal information could be helpful. Shoulder hours could be important for estimating sleep disturbance [15], which would not be captured by $L_{\text {night }}$ and require short-term variations in the map (e.g. 15 -minute $L_{A e q}$ ) to be obtained. On a shorter time scale, percentile levels or event counts could allow estimating the noticeability of environmental sounds.

These aforementioned considerations form the starting point of this work. The aim is to develop more accurate and dynamic maps based on continuous noise monitoring. The term "dynamic noise map" is used to refer to a noise map that is updated in short time intervals on the basis of measurements performed in a dense, yet efficiently spaced network of noise sensors. As a complement to an energy-based noise level indicator such as $L_{A e q}$, the proposed methodology can also include other indicators that could be relevant for the evaluation of the effect of noise on people such as the percentile levels $L_{10}$ and $L_{90}$.

Recently, a procedure for obtaining a dynamic map was developed by assuming that sound propagation is independent of time, allowing to update levels at receivers by only considering temporal source power variations $[16,17]$. However this method fails when meteorological effects cannot be assumed to be constant and inaccuracy in modelled propagation becomes observable. To increase spatial detail, one could use mobile measurement stations equipped with GPS to measure in a grid with high spatial resolution [18]. The challenge in the latter lies in combining spatial and temporal detail [19]. Dynamic maps based on microscopic traffic simulations [20, 21] could resolve only part of the aforementioned issues and are moreover very CPU time costly.

In this paper, a model-based interpolation method to calculate dynamic noise maps on the basis of medium-density noise monitoring networks is proposed. The model not only adapts the sound powers over time, but also accounts for temporal variations in the propagation conditions. Both equivalent levels and percentile noise levels are considered in this work. The basic assumption underlying the model-based interpolation is that there is a reasonably good model for predicting sound indicators in the area under study, even if this model is not very accurate for instantaneous level prediction. Inaccuracy may occur in the emission of the source but also in the calculated propagation. The interpolation will tune the source and propagation on the basis of measurements and in that way improve predictions at locations where no measurements are available.

This article is organized as follows. In section 2, the general layout of the model is introduced. In section 3, the methodology for correcting the source emission levels and propagation related parameters, based on measurements, is presented. In Section 4, the implementation and scalability 
of the model is considered. In section 5, a case study is described using 8 microphones in a city quarter during a 2-month experiment. Finally, conclusions are drawn in section 6 .

\section{General methodology}

The proposed method for dynamic interpolation relies on an underlying source and propagation model. This model has to be sufficiently accurate, but as it will be tuned based on measurements, the highest possible accuracy on source categorisation and propagation path calculation is not required. A careful balance between accuracy and efficiency will be therefore sought. Tuning model parameters based on measurements has to be based on a sufficiently small number of degrees of freedom. The number of degrees of freedom that can be resolved is determined by the number of measurement stations and the assumptions on temporal coherence on the time scale considered in the sources. This section will therefore also focus on reducing the number of degrees of freedom.

\section{$2.1 \quad$ Underlying model}

\subsubsection{Temporal aggregation}

It is obvious that models aiming at predicting instantaneous sound pressure levels, for example on a second-by-second basis, $L_{A e q, 1 s e c}$, would most likely fail. Moreover, even if interpolation between monitoring stations at a second-by-second basis were possible, the information obtained would be very hard to interpret. Thus a suitable aggregation period has to be chosen. This requires a careful balance between stability and repeatability at the one hand, and a sufficient degree of dynamics at the other hand. In an urban environment, short-term dynamics are mainly determined by passages of motorized vehicles [20]. A suitable aggregation period depends on the traffic intensity, but 10 to 15 minutes is usually chosen as an appropriate monitoring period. Sequential monitoring over such time intervals results in low standard errors between measurement periods for continuous traffic flows, and still includes enough temporal resolution to capture typical (diurnal) activity patterns [22]. Finally, one should also consider the latency in calculating updated maps. The larger the temporal step, the larger the latency. Based on these considerations, a 15-minute temporal interval was chosen to produce the dynamic noise maps.

The equivalent sound pressure level $L_{A e q, 15 \min }$ includes the total sound energy received at a measurement location. Every sound source, fixed or mobile, contributes an amount to this quantity independently. Moreover, the total energy over 15 minutes determines the $L_{\text {Aeq }}$ independently of the emission instant in time. In addition, it is a preferred indicator for noise mapping. Next to $L_{\text {Aeq }}$, percentile noise levels $L_{N}$ are also of importance when analysing the soundscape. The level $L_{10}$ indicates the noise level exceeded $10 \%$ of the measurement time, and is therefore indicative for peak levels. The level $L_{90}$ indicates the noise level exceeded $90 \%$ of the measurement time, and can be considered as the background noise level. Both $L_{10}$ and $L_{90}$ are widely used in studies of noise disturbance and background noise quality. Although the model will be developed for $L_{A e q}, L_{10}$ and $L_{90}$, it can readily be extended to other indicators. 


\subsubsection{Model for predicting $L_{e q}$}

The model for $L_{e q}$ calculates the total contribution from all sources at point $p$ and time $t$, for frequency $f$ :

$$
L_{e q, f}(p, t)=10 \log _{10} \sum_{i}^{N_{s}} \sum_{j}^{N_{h}} 10^{0.1\left[L_{W, f, i}^{\prime}(t)-A_{f, i, j}^{\prime}(p, t)\right]}
$$

where the sources are labelled by index $i$ and the propagation paths are labelled by index $j$, which allows for different paths between the $i^{t h}$ source and location $p ; N_{s}$ is the number of sources and $N_{h}$ is the total number of propagation paths; the primes stress that the initially estimated value is modified based on measurements.

Actual values for sound emission $L_{W, f, i}^{\prime}$ and attenuation $A_{f, i, j}^{\prime}$ are obtained from their initially estimated value $L_{W, f, i}$ and $A_{f, i, j}$ and the measured levels $L_{x, f, m e a s}(p, t-1)$. For this, it is assumed that the actual value deviates only by a "small" amount:

$$
\begin{gathered}
L_{W, f, i}^{\prime}(t)=L_{W, f, i}(t)+\epsilon_{f, i}^{t-1} \\
A_{f, i, j}^{\prime}(p, t)=A_{f, i, j}(p)+\delta_{f, i, j}^{t-1}
\end{gathered}
$$

where, $L_{W, f, i}(t)$ is the source power level at time $t . L_{W, f, i}(t)$ can be calculated by a source model or it can be obtained from a close-proximity measurement. Both $\epsilon_{f, i}^{t-1}$ and $\delta_{f, i, j}^{t-1}$ are functions of frequency $f$ and time $t$, but can be functions of several other parameters as well. Note that no explicit dependence of the initially estimated attenuation on time is assumed, and thus any variation that might occur will need to be captured by the correction term. Extracting $\epsilon_{f, i}^{t-1}$ and $\delta_{f, i, j}^{t-1}$ from the measurements $L_{x, f, \text { meas }}(p, t-1)$ will be done by the least mean squares method including restrictions on the size of $\epsilon_{f}$ and $\delta_{f}$.

\subsubsection{Model for predicting $L_{10}$ and $L_{90}$}

Fluctuations in noise levels can occur for different reasons: a spatially static sound sources can show a clear temporal pattern (e.g. a cooling unit switching on and off), a source could move, or the propagation conditions from a spatially static source can change quickly, e.g. due to wind gusts. The latter type of fluctuation will not be considered here. For spatially fixed sources with fluctuating emission, the relationship between equivalent noise level and percentile noise levels is directly determined by the source. However, for moving sources, distance and speed have a strong influence on this relationship. In this work we will mainly consider road traffic as the moving sources. Rail and air traffic often only influence the low indexed percentile levels and can therefore easily be added.

A full blown microscopic traffic simulation allows to calculate percentile noise levels quite accurately [20, 21, 23, 24]. However, it also requires many parameters that do not relate to measured noise levels in a straightforward way. Steering microscopic traffic simulation from roadside noise measurements in real-time is still beyond the possibilities of today's computers, in particular when it has to be applied to a whole city. Therefore an alternative approach is followed.

The main assumptions behind this approach are (i) that the fluctuation in noise level due to road traffic is mainly caused by the traffic on the closest road, whereas more distant traffic only contributes to a relatively constant background hum, and (ii) that the relationship between $L_{A e q}$, 
$L_{10}$ and $L_{90}$ is mainly influenced by the distance to this road, and by the traffic flow rate, the vehicle speed and the amount of heavy vehicles on this closest road. A simplified simulation model can then be used to estimate these relationships, based on the simulated temporal envelope of the sound level near a single road carrying free flow traffic [25]. For this, a $2 \mathrm{~km}$ stretch of a straight road was modelled, on which vehicles were loaded at one end, which then travelled to the other end. The number of vehicle passby's per time unit followed a Poisson distribution, which is typical for free flow traffic, containing platoons of various lengths. The emission of each vehicle was calculated using the Harmonoise/Imagine road traffic noise emission model, and free field propagation was used to calculate the instantaneous sound level at a receiver point next to the road in the middle of the $2 \mathrm{~km}$ segment. From the instantaneous sound level that was simulated at the location of the receiver, the relationship between $L_{A e q}, L_{10}$ and $L_{90}$ was derived, and was tabulated for a range of scenarios in which vehicle speed, percentage of heavy vehicles, distance to the road and traffic flow were varied.

Within the dynamic noise mapping approach followed in this paper, for each receiver, the distance to the closest road segment is determined and the contribution of this road to $L_{A e q}$ is extracted. Based on the traffic intensity and speed limit on this road, corresponding values for $L_{10}$ and $L_{90}$ are then extracted from the pre-calculated tables. For contributions to the traffic noise level that follow paths that contain multiple reflections, diffraction over buildings, or scattering on atmospheric turbulence, it is assumed that peaks in the sound level smooth out and consequently that $L_{10}, L_{\text {Aeq }}$, and $L_{90}$ are almost equal. For those situations in which the receiver is close to more than one road, multiple contributions will need to be added. As the distribution of the sound level caused by each road separately is not known, a more elaborate derivation has to be made to obtain estimates of $L_{10}$ and $L_{90}$. Details on this procedure can be found in Section A.1.

\subsection{Reducing the number of degrees of freedom}

From Equations 1, 2 and 3, it is clear that the number of unknown $\epsilon$ is $N_{s}$ and the number of unknown $\delta$ is $N_{s} \cdot N_{h}$ for each immission point, which makes the system of equations, obtained from matching calculations and a limited number of sound observations, to be solved strongly under-determined. Moreover, many sources and propagation paths have only a very small influence on each immission point, which makes the system ill-conditioned. Additionally, for every update of the map, the double sum in Equation 1 has to be recalculated, which amounts to $N_{s} \cdot N_{h} \cdot N$ calculations, where $N$ is the number of measurement stations.

To limit the size of the sum over all $N_{s}$ sources and the degrees of freedom in $\epsilon$, sources need to be categorized. Categorization and grouping is particularly useful for sound sources that are spread over large areas such as road traffic noise. Categorization of road traffic noise sources could depend on traffic intensity, speed limit, or other categorization methods, such as grouping sources to highways, major city roads and minor city roads.

In the underlying noise mapping model, three categories of propagation paths are included for road traffic:

- the horizontal (2D) path, consisting of direct line-of-sight propagation and reflections or diffractions in the horizontal plane (near vertical edges);

- the vertically diffracted path, i.e. the contribution caused by multiple reflections and diffractions over roof tops (excluding the horizontal plane) [10]; 
- the scattered path, i.e. the contribution to the immission due to turbulence scattering, which is mainly important in shielded areas [26].

It is essential to keep corrections on the three above mentioned categories of propagation paths separately. It is expected that the horizontal path is estimated quite accurately, the vertical path could be slightly inaccurate yet not time dependent, whereas the scattered path may change significantly over time.

Although several possible propagation paths have been grouped in the above three categories, $\delta$ could still depend on the positions of source and receiver. In this way, the correction term $\delta$ still contains a large number of degrees of freedom. Two assumptions are made to further reduce the number of degrees of freedom: (i) it is assumed that the source corrections are identical in the same source category; the source correction $\epsilon_{f, i}$ then becomes $\epsilon_{f, m}$, where $i$ is a source and $m$ is a source category; (ii) it is assumed that the propagation corrections are also identical in the same source category and for the same category of propagation paths. The propagation correction $\delta_{f, i, j}$ then becomes $\delta_{f, m, n}$, where $m$ is a source category and $n$ is a propagation path category. Based on these categorizations, Equation 1 can be rewritten as follows:

$$
L_{e q, f}(p, t)=10 \log _{10}\left\{\sum_{n=1}^{N_{n}} \sum_{j \in n} \sum_{m=1}^{N_{m}} \sum_{i \in m} 10^{0.1\left[L_{W, f, i}^{\prime}(t)-A_{f, i, j}^{\prime}(p, t)\right]}\right\}
$$

where $i \in m$ refers to $i$ belonging to the source category $m$ and $j \in n$ refers to $j$ belonging to the propagation category $n . N_{m}$ is the total number of source categories and $N_{n}$ is the total number of propagation path categories. The corrections for the same source or propagation path category are supposed to be equal. Consequently, the corrections mentioned in Equations 2 and 3 can be moved outside the summation for the same category. The formula then changes to:

$$
L_{e q, f}(p, t)=10 \log _{10}\left\{\sum_{m=1}^{N_{m}} 10^{0.1 \epsilon_{f, m}^{t}} \sum_{n=1}^{N_{n}} 10^{-0.1 \delta_{f, m, n}^{t}} \sum_{j \in n} \sum_{i \in m} 10^{0.1\left[L_{W, f, i}(t)-A_{f, i, j}(p, t)\right]}\right\}
$$

The double sum $\sum_{j \in n} \sum_{i \in m} 10^{0.1\left[L_{W, f, i}(t)-A_{f, i, j}(p, t)\right]}$ indicates a separate noise map, calculated for source category $m$ and propagation category $k$, denoting the total contribution of a source category by a propagation category. In this way, the most time-consuming part of the dynamic noise map calculation can be performed and stored in advance, which saves updating time considerably. Next to this, the number of degrees of freedom reduces from $N_{s}+N_{s} \cdot N_{h} \cdot N$ to $N_{m}+N_{m} \cdot N_{n} \cdot N$, with $N$ the number of measurement stations.

The equations for the underlying model and the reduction of the number of degrees of freedom explicitly depend on frequency, for example in 1/3-octave bands. Although there are no fundamental constraints on performing the fit to measurements on a frequency (band) per frequency (band) basis, this additional degree of freedom jeopardizes the uniqueness of the solution, and thus could easily lead to over-fitting. Therefore, in this work it is assumed that the traffic source spectrum is reasonably accurate, and that the spectrum of atmospheric attenuation is reasonably accurate. In that case, it can be assumed that the corrections on both source sound power and propagation path attenuation are independent of frequency. For industrial noise sources, explicitly including frequency dependent corrections is more appropriate, unless sound power spectra are explicitly obtained from a separate measurement. 


\section{Correction terms for sources and propagation paths}

The correction terms $\epsilon$ and $\delta$ are obtained by minimizing the squared error between predictions and measurements:

$$
e=\sum_{p}^{N}\left(\sum_{f}\left[L_{e q, f}(p, t)-L_{e q, f, \text { meas }}(p, t)\right]^{2}+\sum_{x}\left[L_{x}(p, t)-L_{x, \text { meas }}(p, t)\right]^{2}\right)
$$

where $p$ is the measurement position; $x$ is the percentile indicator (either 10 or 90 in this publication), and $f$ is the frequency. As shown in Equation 6, the frequency dependency of $L_{e q}$ is explicitly taken into account, while this is not the case for the percentile levels where only the overall A-weighted level is used. The main reason for this is that the spectrum of percentile levels is ill-defined, yet one could think of a useful extension of the algorithms in this direction. Assuming that Equation 6 has a unique minimum, this minimum can be obtained by setting the partial derivatives with respect to the variables $\epsilon$ and $\delta$ of the sum in Equation 6 equal to zero; $\epsilon$ and $\delta$ can then be resolved. In this publication, a slightly different approach is followed.

Inspired by the least mean squares algorithm (LMS) that is popular in signal processing, Equation 6 is not minimized explicitly at every time step, but on average over a longer time period, hereby assuming that $\epsilon$ and $\delta$ change only slowly with time. As in the signal processing case, it is then assumed that the minimum in the error function will be reached by taking small steps in the direction of the minimum, taking into account only the instantaneous values in calculating the derivative. Note that in situations where the LMS algorithm is used in signal processing, the uniqueness of the solution and convergence can be proven, yet this is not the case here. Application of the algorithm will show to what extent convergence can be reached. The correction of source strength, $\epsilon$, can be updated in the following way:

$\epsilon_{m}^{t} \approx \epsilon_{m}^{t-1}-\frac{\partial}{\partial \epsilon_{m}^{t-1}}\left\{\mu_{\epsilon} \sum_{p}^{N}\left(\sum_{f}\left[L_{e q, f}(p, t)-L_{\text {eq,f,meas }}(p, t)\right]^{2}+\sum_{x}\left[L_{x}(p, t)-L_{x, \text { meas }}(p, t)\right]^{2}\right)+\beta_{\epsilon}^{\prime}\left(\epsilon_{m}^{t-1}\right)^{2}\right\}$

where $\mu_{\epsilon}$ is a small number, and $\beta_{\epsilon}^{\prime}$ is a regularisation constraint that keeps $\epsilon_{m}^{t}$ small. Merging the constant into $\beta_{\epsilon}$, Equation 7 changes to:

$$
\begin{aligned}
\epsilon_{m}^{t} & =\epsilon_{m}^{t-1}\left(1-\beta_{\epsilon}\right)-\mu_{\epsilon} \sum_{p}^{N}\left\{\sum_{f} 2\left[L_{e q, f}(p, t)-L_{e q, f, \text { meas }}(p, t)\right] \frac{\partial}{\partial \epsilon_{m}^{t-1}} L_{e q, f}(p, t)\right. \\
& \left.+\sum_{x} 2\left[L_{x}(p, t)-L_{x, \text { meas }}(p, t)\right] \frac{\partial}{\partial \epsilon_{m}^{t-1}} L_{x}(p, t)\right\}
\end{aligned}
$$

In a similar way, one obtains the following equation for the correction of the propagation path attenuation:

$$
\begin{aligned}
\delta_{m, n}^{t} & =\delta_{m, n}^{t-1}\left(1-\beta_{\delta}\right)-\mu_{\delta} \sum_{p}^{N}\left\{\sum_{f} 2\left[L_{e q, f}(p, t)-L_{e q, f, \text { meas }}(p, t)\right] \frac{\partial}{\partial \delta_{m, n}^{t-1}} L_{e q, f}(p, t)\right. \\
& \left.+\sum_{x} 2\left[L_{x}(p, t)-L_{x, \text { meas }}(p, t)\right] \frac{\partial}{\partial \delta_{m, n}^{t-1}} L_{x}(p, t)\right\}
\end{aligned}
$$




\subsection{Source correction $\epsilon$}

To quantify the contribution of $L_{e q}$ to the correction for source category $m$, the derivatives $\frac{\partial}{\partial \epsilon_{m}^{t-1}} L_{e q, f}(p, t)$ and $\frac{\partial}{\partial \epsilon_{m}^{t-1}} L_{x}(p, t)$ need to be solved. Substituting Equation 5 into the derivatives, and using the technique of derivative of implicit functions, leads to:

$$
\begin{aligned}
\frac{\partial}{\partial \epsilon_{m}^{t-1}} L_{e q, f}(p, t)= & \frac{10}{\ln 10} \frac{1}{10^{0.1 L_{e q, f}(p, t)}} \\
& \frac{\partial}{\partial \epsilon_{m}^{t-1}}\left\{\sum_{m^{\prime}=1}^{N_{m}} 10^{0.1 \epsilon_{m^{\prime}}^{t-1}} \sum_{n=1}^{N_{n}} 10^{-0.1 \delta_{m^{\prime}, n}^{t-1}} \sum_{j \in n} \sum_{i \in m^{\prime}} 10^{0.1\left[L_{W, f, i}(t)-A_{f, i, j}(p, t)\right]}\right\}(10)
\end{aligned}
$$

Since the corrections for different source categories are considered to be independent from each other, the derivatives for other categories $\left(m^{\prime} \neq m\right)$ with respect to $\epsilon_{m}^{t-1}$ will vanish, which means that $\frac{\partial}{\partial \epsilon_{m}^{t-1}} \sum_{m^{\prime}=1}^{N_{m}} 10^{0.1 \epsilon_{m^{\prime}}^{t-1}}=\frac{\partial}{\partial \epsilon_{m}^{t-1}} 10^{0.1 \epsilon_{m}^{t-1}}$. Equation 10 is then simplified as follows:

$$
\frac{\partial}{\partial \epsilon_{m}^{t-1}} L_{e q, f}(p, t)=\frac{10^{0.1 \epsilon_{m}^{t-1}} \sum_{n=1}^{N_{n}} 10^{-0.1 \delta_{m, n}^{t-1}} \sum_{j \in n} \sum_{i \in m} 10^{0.1\left[L_{W, f, i}(t)-A_{f, i, j}(p, t)\right]}}{10^{0.1 L_{e q, f}(p, t)}}
$$

The following explains Equation 11 in more detail:

- $L_{W, f, i}(t)$ is the power level of the $i^{t h}$ source, which may be calculated by the source power model;

- $\sum_{j \in n} \sum_{i \in m} 10^{0.1\left(L_{W, f, i}(t)-A_{f, i, j}(p, t)\right)}$ is the sound power originating from all sources within the same category to the measurement position $p$, through all propagations paths within the $n^{\text {th }}$ category, where $n=1$ is the horizontal sound path, $n=2$ is the vertical sound path and $n=3$ is the scattered sound path;

- $10^{0.1 \epsilon_{m}^{t-1}} \sum_{n=1}^{N_{n}} 10^{-0.1 \delta_{f, m, n}^{t-1}} \sum_{j \in n} \sum_{i \in m} 10^{0.1\left[L_{W, f, i}(t)-A_{f, i, j}(p, t)\right]}$ is the sound power originating from all sources within the same category to the measurement position $p$, through all propagation paths. This value includes the source correction term $\epsilon_{m}^{t-1}$ and the propagation correction term $\delta_{m, n}^{t-1}$;

- $10^{0.1 L_{e q, f}(p, t)}$ is the total equivalent energy which can be calculated by Equation 5 .

In a similar way, the derivatives $\frac{\partial}{\partial \epsilon_{m}^{t-1}} L_{x}(p, t)$ can be derived. However, $L_{10}$ and $L_{90}$ cannot be calculated in the same straightforward as $L_{e q, f}$. Details on the calculation of $L_{10}, L_{90}$ and their derivative to $\epsilon_{m}^{t-1}$ and $\delta_{m, n}^{t-1}$ can be found in Section A.2. 
Using Equations 8 and 11, and putting the constants into a fitting parameter, the correction term $\epsilon$ for a specified source category $m$ becomes:

$$
\begin{aligned}
\epsilon_{m}^{t}= & \epsilon_{m}^{t-1}\left(1-\beta_{\epsilon}\right)-\mu_{\epsilon} \sum_{p}^{N}\left\{\left[L_{e q, f}(p, t)-L_{\text {eq, }, \text { meas }}(p, t)\right]\right. \\
& \frac{10^{0.1 \epsilon_{m}^{t-1}} \sum_{n=1}^{N_{n}} 10^{-0.1 \delta_{m, n}^{t-1}} \sum_{j \in n} \sum_{i \in m} 10^{0.1\left[L_{W, f, i}(t)-A_{f, i, j}(p, t)\right]}}{10^{0.1 L_{\text {eq }, f}(p, t)}} \\
& \left.+\left(L_{10}-L_{10, \text { meas }}\right) \frac{\partial}{\partial \epsilon_{m}^{t-1}} L_{10}+\left(L_{90}-L_{90, \text { meas }}\right) \frac{\partial}{\partial \epsilon_{m}^{t-1}} L_{90}\right\}
\end{aligned}
$$

\subsection{Propagation path correction $\delta$}

The LMS method is also used to calculate $\delta_{m, n}^{t}$. A similar procedure is applied as with the calculation of $\epsilon_{m}^{t}$. Also, note that the corrections for different propagation paths are independent, meaning that the derivatives to paths other than $n$ will vanish. The derivative can be simplified to:

$$
\begin{aligned}
\frac{\partial}{\partial \delta_{m, n}^{t-1}} L_{e q, f}(p, t)= & \frac{10}{\ln 10} \frac{1}{10^{0.1 L_{e q, f}(p, t)}} \\
& \frac{\partial}{\partial \delta_{m, n}^{t-1}}\left\{\sum_{m^{\prime}=1}^{N_{m}} 10^{0.1 \epsilon_{m^{\prime}}^{t-1}} \sum_{n^{\prime}=1}^{N_{n}} 10^{-0.1 \delta_{m^{\prime}, n^{\prime}}^{t-1}} \sum_{j \in n^{\prime}} \sum_{i \in m^{\prime}} 10^{0.1\left[L_{W, f, i}(t)-A_{f, i, j}(p, t)\right]}\right\} \\
= & -\frac{10^{0.1 \epsilon_{m}^{t-1}} 10^{-0.1 \delta_{m, n}^{t-1}} \sum_{j \in n} \sum_{i \in m} 10^{0.1\left[L_{W, f, i}(t)-A_{f, i, j}(p, t)\right]}}{10^{0.1 L_{e q, f}(p, t)}}
\end{aligned}
$$

According to Equations 9 and 13, the correction term $\delta$ for a specified source category $m$ and propagation path category $n$ becomes:

$$
\begin{aligned}
\delta_{m, n}^{t}= & \delta_{m, n}^{t-1}\left(1-\beta_{\delta}\right)-\mu_{\epsilon} \sum_{p}^{N}\left\{-\left[L_{e q, f}(p, t)-L_{e q, f, \text { meas }}(p, t)\right]\right. \\
& \frac{10^{0.1 \epsilon_{m}^{t-1}} 10^{-0.1 \delta_{m, n}^{t-1}} \sum_{j \in n} \sum_{i \in m} 10^{0.1\left[L_{W, f, i}(t)-A_{f, i, j}(p, t)\right]}}{10^{0.1 L_{e q, f}(p, t)}} \\
& \left.+\left(L_{10}-L_{10, \text { meas }}\right) \frac{\partial}{\partial \delta_{m, n}^{t-1}} L_{10}+\left(L_{90}-L_{90, \text { meas }}\right) \frac{\partial}{\partial \delta_{m, n}^{t-1}} L_{90}\right\}
\end{aligned}
$$

The details of solving $\frac{\partial}{\partial \delta_{m, n}^{t-1}} L_{10}$ and $\frac{\partial}{\partial \delta_{m, n}^{t-1}} L_{10}$ can be found in Section A.2.

\section{Implementation and scalability}

Figure 1 summarizes the calculation flow of the model. In a first initialization stage, propagation path attenuations are calculated and stored, for the horizontal, vertical and scattered path categories, and for all categorized sources, the correction terms are initialized to zero, and an initial map is calculated, according to Equation 5. Subsequently, the map is iteratively updated based 


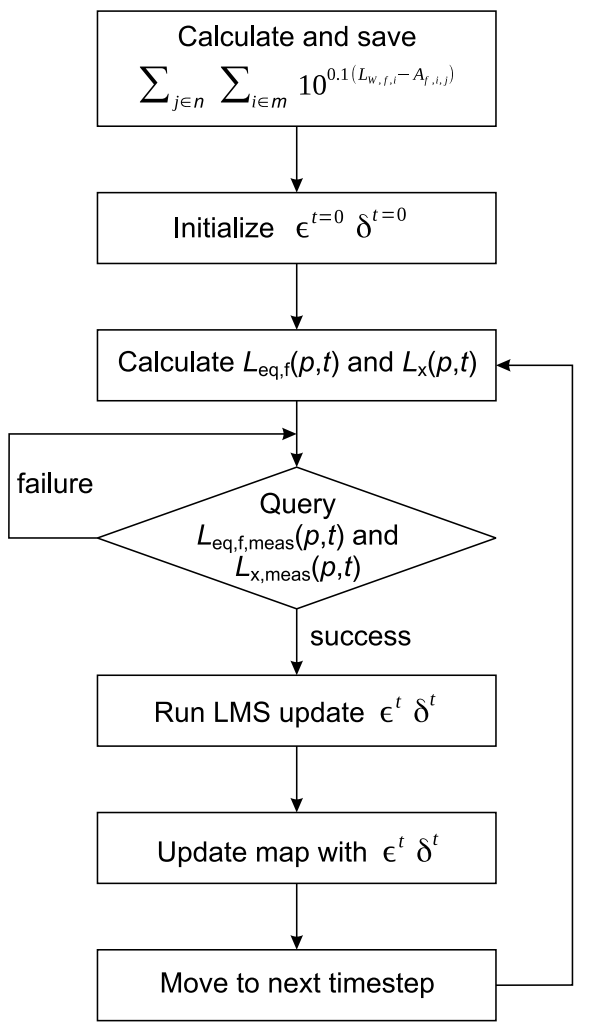

Figure 1: Calculation flow of the model.

on measurements, queried from a database. Once measurements are available, the LMS update algorithm is run in order to obtain corrected values for $\epsilon$ and $\delta$, a new map is generated and the timestep is increased (in the present work with 15 minutes). Note that using this method, the maps $\sum_{j \in n} \sum_{i \in m} 10^{0.1\left(L_{W, f, i}(t)-A_{f, i, j}(p, t)\right)}$ are calculated and stored in advance.

The number of measurement stations is a limiting factor for the scalability of the model to city-wide areas. However, the proposed methodology includes two mechanisms to facilitate this process. Firstly, the most time-consuming part of the model is formed by the calculation of the propagation attenuations. The computational complexity of this part clearly depends on the size of the area considered, but this calculation has to be carried out only once, at the start of the updating cycle. For calculating the propagation paths in the horizontal plane, a ray/beam tracing method is recommended, such as the algorithm applied in [23], whereas highly efficient methods to calculate the vertical and scattered propagation paths have been developed recently in the framework of the QSIDE project [10], speeding up this initial calculation step. Once the initial propagation calculations are available, dynamically updated maps are obtained by weighted addition of the series of initial maps, according to Equation 5. The latter operation is relatively fast.

Secondly, the reduction of the number of degrees of freedom (Section 2.2) is an essential step in the process, whereby the complexity of the model can be matched with the number of measurement stations available. Obviously, the more measurement stations available, the finer-grained the source and propagation path grouping can be, and thus the more dynamic effects that can be taken into account, leading to an increasingly better prediction. However, even with a few, well-placed mea- 
surement stations, already a significant improvement over the static map can be obtained, as will be demonstrated in Section 5. For larger areas under consideration, it is therefore not necessary to cover the whole area with a fine-resolution grid of measurement stations; instead, it is more important to have a good coverage of each source category that is taken into account. Thus, the number of sensors required does not scale with the size of the city area under consideration.

\section{Case study}

\subsection{Overview}

The Katendrecht district of Rotterdam, the Netherlands, is considered to validate the proposed dynamic mapping model. This district is located on a peninsula with an area of $1.18 \mathrm{~km}^{2}$ and with 4445 residential inhabitants [27]. The main noise source in the case study area is formed by road traffic during daytime. During nighttime, industrial sources to the south of the peninsula may become important. The grouping of noise sources assumes similar uncertainty on the accuracy of the source data within one group. Inaccuracy can be attributed to traffic information or emission data for individual vehicles. The latter in turn depends on the fleet composition (including tires and maintenance) and on the road surface. Fleet maintenance, the state of tires, etc. is expected to be relatively constant over a whole city. Road surface and road maintenance state may differ amongst neighbourhoods or districts. But for the area under study, which is relatively small and homogeneous, it can be assumed similar for the whole area. Hence, in this particular case study, there is no need to categorize the traffic sound sources depending on their location. Uncertainty on traffic information and its effect on sound emission often depends on traffic intensity. Highway traffic and traffic on main arterial roads is known with much higher accuracy than traffic on local access roads for example. Therefore, traffic sources are grouped into four categories, based on traffic intensity or sound power level used in the calculation of strategic noise maps; the latter can be considered as a proxy for the former. Sound power levels caused by road traffic are available from strategic noise mapping for every $10 \mathrm{~m}$ stretch of road. Since the sound power levels of the traffic sources are not heavy-tailed distributions, categorization is performed by using the Jenks-naturalbreaks classification method [28]. This method seeks to minimize the variance inside a group and to maximize the variance between groups:

$$
\begin{aligned}
& \min \left\{\sum_{a=u}^{v}\left(A[a]-\text { mean }_{u, v}\right)^{2}\right\} \\
& \max \left\{\sum_{b=t}^{s}\left(\text { mean }_{t}-\text { mean }_{b}\right)^{2}\right\}
\end{aligned}
$$

The available traffic data does not contain traffic information on a 15-minute resolution. Therefore, diurnal patterns for traffic are extracted from event counts in a couple of sensor nodes that were placed close to the roadside. A similar diurnal pattern is assumed for all other roads. The industrial sources are considered as a separate source category, which are represented by 4 separate

point sources with the same source power spectrum. An estimate for the spectrum of the source power of industrial sources is determined from a far-field measurement. The categorized spectra are shown in Figure 2 as $L_{W \text { Aday }}, L_{W}$ Aevening and $L_{W}$ Anight, respectively. 

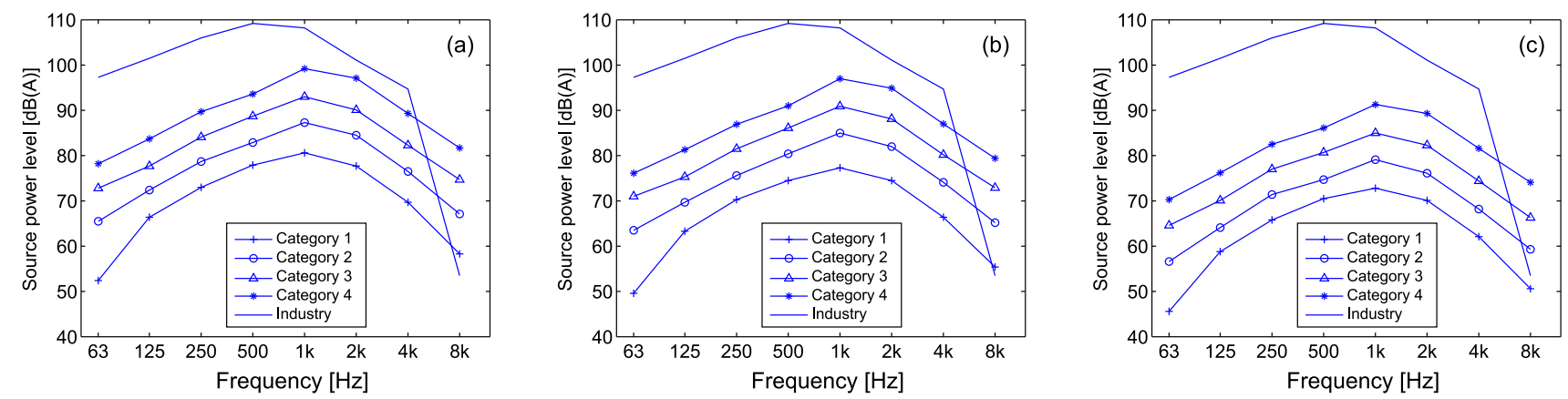

Figure 2: Spectra of the categorized road traffic noise and industry noise sources: (a) $L_{W A \text {,day }}$, (b) $L_{W A, \text { evening, }}$ (c) $L_{W A, \text { night }}$.
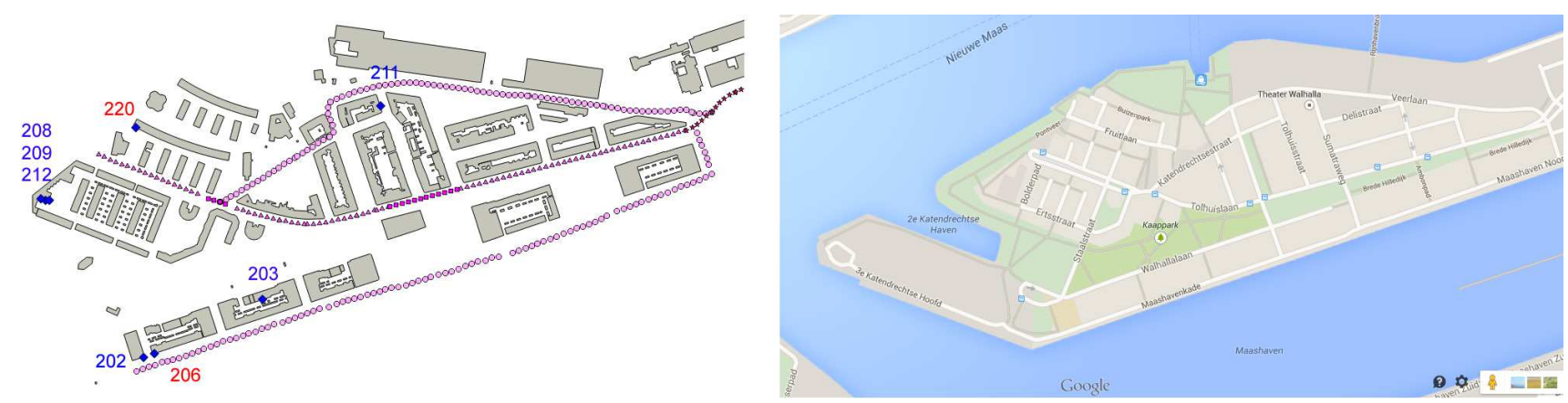

Figure 3: Overview of the case study area. (left) Buildings and measurement stations inside the case study area. The validation positions are shown in red with ID's 206 and 220. Road traffic source categories are indicated by circles (category 1), triangles (category 2), squares (category 3 ) and stars (category 4). (right) Overview of the validation site (Google maps).

Eight measurement stations [29] are placed on buildings in the peninsula, as shown in Figure 3. Six of these are used to calculate the source correction $\epsilon$ and propagation correction $\delta$. The two remaining measurement stations are used to validate the model. In this case study, $L_{A e q}$ (including spectrum information), $L_{10}$ and $L_{90}$ is extracted on a 15-minute basis from the measurement stations to create the dynamic map.

Partial noise maps are calculated for the horizontal path [30], the vertical path [10] and the scattered path [26] for each of the 5 source categories. When calculating the horizontal path, only 1 reflection and 1 diffraction on vertical edges (horizontal plane) are taken into account and the buildings are assumed to have a reflection coefficient of 0.95 . When calculating the vertical path, only sources within $1500 \mathrm{~m}$ from the receiver are considered. When calculating the scattered path, the structure parameters of velocity and temperature are $C_{v}^{2}=0.01$ and $C_{t}^{2}=0.002$, respectively. For the vertical and scattered paths, the reflections in the vertical plane are explicitly included, where it is assumed that the length of the street is much longer than the height of the buildings. However, for real measurements, the coherence may be strong when the measurement position is close to a reflector. In this case study, the measurement stations are often placed on the façade of buildings. Therefore a correction of $-3 \mathrm{~dB}$ is applied to the measurements, in order to avoid the 

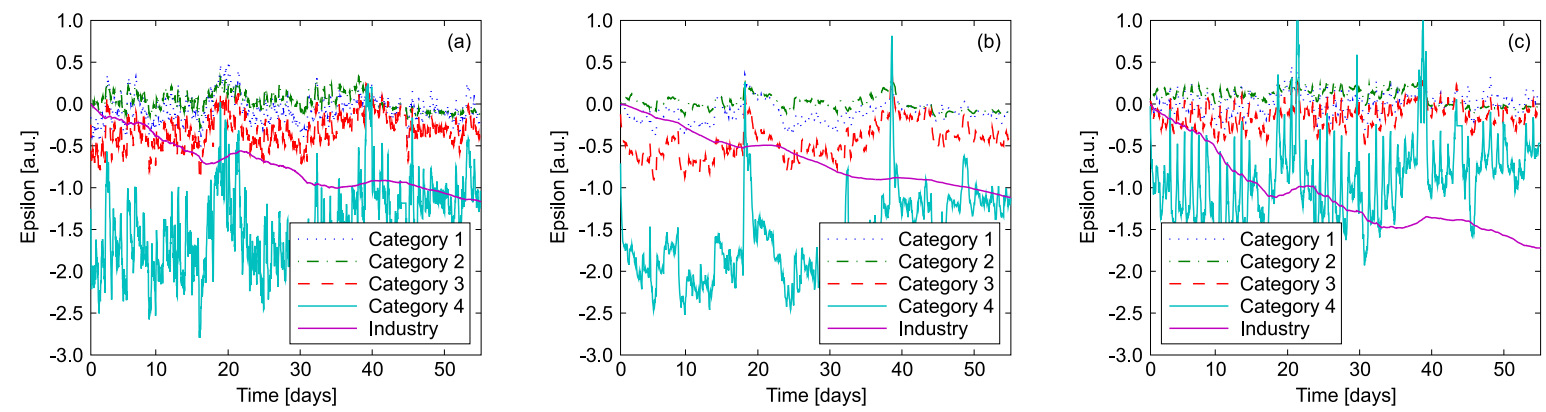

Figure 4: Time history of the emission correction $\epsilon$, over the course of 55 days, for all categories of sources; (a) day period, (b) evening period, (c) night period.
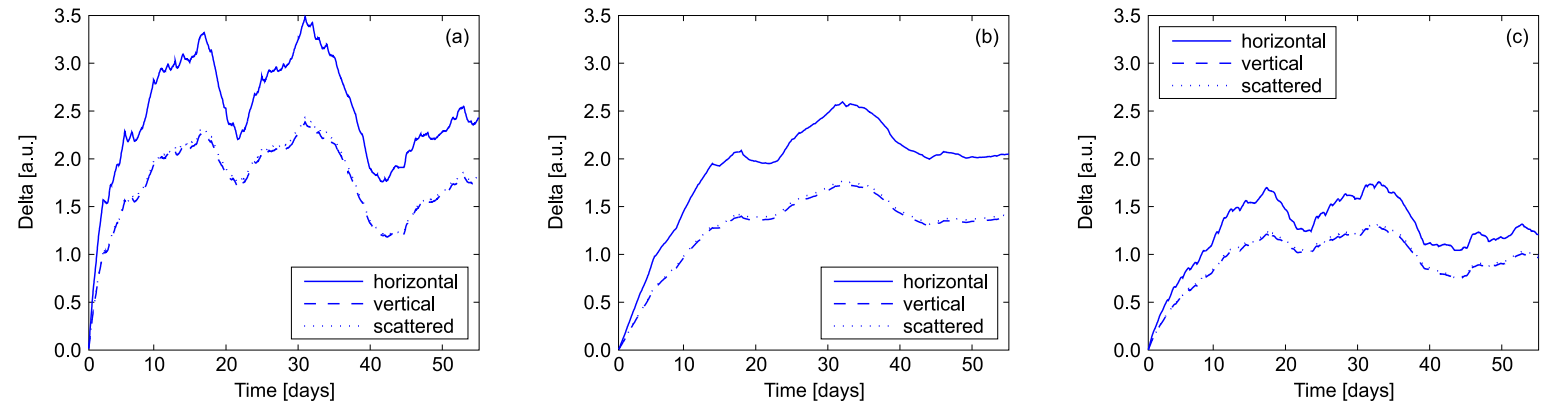

Figure 5: Time history of the propagation correction $\delta$, over the course of 55 days, for the traffic sources of category 1, for the three propagation path categories considered; (a) day period, (b) evening period, (c) night period.

effect of coherence between the direct and reflected sound [10,31, 32].

\subsection{Analysis of fitting parameters}

Based on the measured and predicted noise levels, and according to the procedure outlined in the above sections, the correction coefficients $\epsilon$ and $\delta$ are calculated, and their time history over a period of 55 days is shown in Figures 4 to 9. The corrections $\epsilon$ and $\delta$ are displayed separately for day, evening and night periods. This is required because, on the one hand, the LMS procedure slowly adapts the corrections to more suitable values, and, on the other hand, the input data for road traffic has fixed values for day, evening, and night periods. Moreover, one can expect that the corrections are more similar between subsequent nights than between day and night periods, as traffic sound shows a clear diurnal pattern. In other words, there is more similarity between the uncertainty in noise emission at 4am on Monday and 4am on Tuesday than there is between 4am and 8am on Tuesday, as an example.

In general, the results show that the source power is overestimated (corrections are mainly negative) and the attenuations are underestimated (corrections are mainly positive). Additionally, the correction during the day periods are greater than during the evening and night periods. Sources from category 1 are closest to most measurement positions, but their strength is low. Therefore, it seems reasonable that the corrections along the horizontal paths are more important than the cor- 

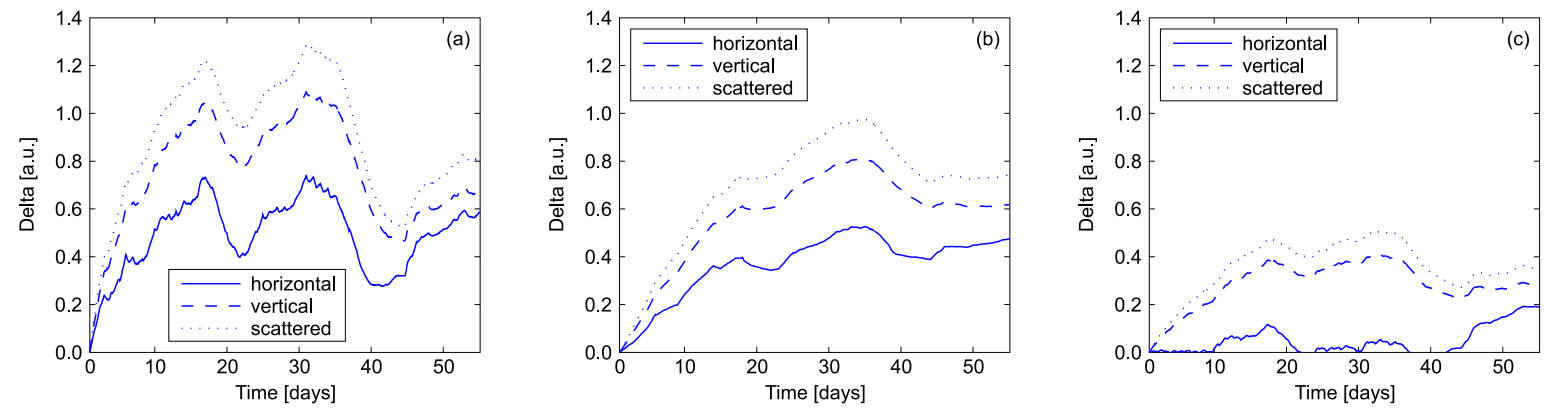

Figure 6: Time history of the propagation correction $\delta$, over the course of 55 days, for the traffic sources of category 2, for the three propagation path categories considered; (a) day period, (b) evening period, (c) night period.
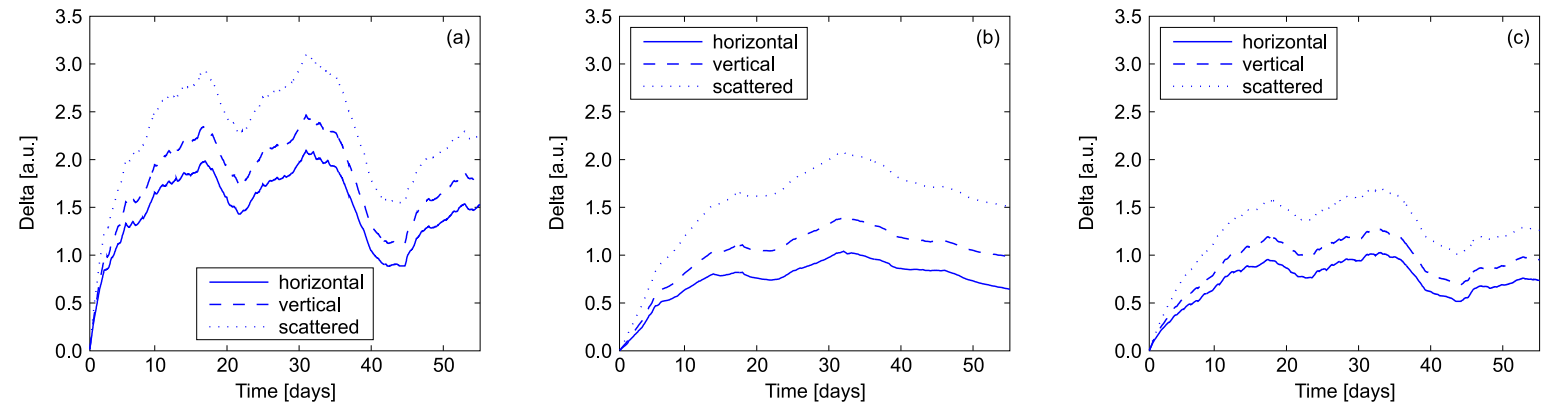

Figure 7: Time history of the propagation correction $\delta$, over the course of 55 days, for the traffic sources of category 3 , for the three propagation path categories considered; (a) day period, (b) evening period, (c) night period.
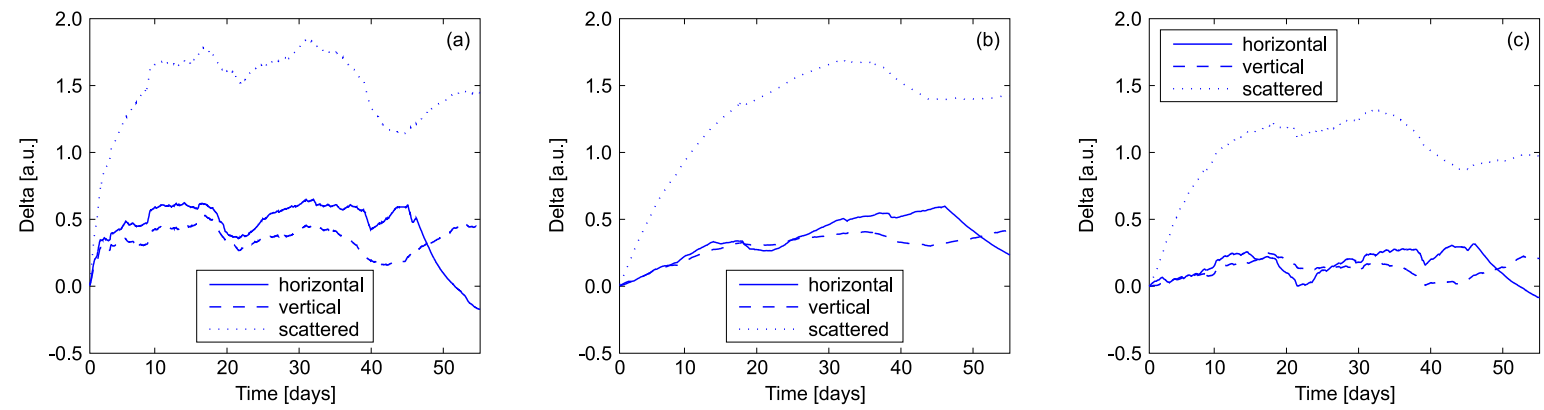

Figure 8: Time history of the propagation correction $\delta$, over the course of 55 days, for the traffic sources of category 4, for the three propagation path categories considered; (a) day period, (b) evening period, (c) night period. 

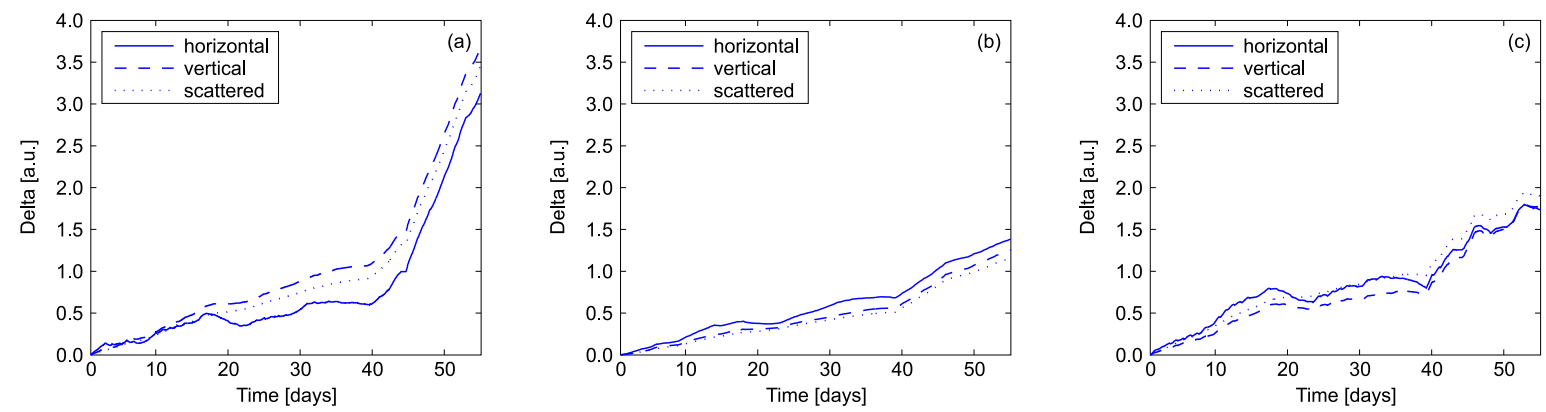

Figure 9: Time history of the propagation correction $\delta$, over the course of 55 days, for the industry sources, for the three propagation path categories considered; (a) day period, (b) evening period, (c) night period.

rections along the vertical and scattered paths, as shown in Figure 5, where the $\delta$ of the horizontal path is above the other two paths. Roads from category 2 are situated further away from most of the fitting positions and their source power is low. This category then has a very limited contribution to the sound level. Figures 4 and 6 show that the source correction $\epsilon$ and propagation correction $\delta$ are consequently very small. The effect of roads from category 3 and category 4 is stronger than those from category 2, as shown in Figures 7 and 8. These sources are mostly screened, so only small adaptations along the horizontal path are expected. The overestimation of the scattered path is probably caused by the strong turbulence strength. The horizontal and vertical path calculations are fairly correct. According to Equations 11 and 13, the correction of $\epsilon$ depends on the contribution of all propagation paths in one specific category, whereas the correction of $\delta$ only depends on the contribution of a specific propagation path in one specific category. Therefore, it can be expected that the source corrections change more quickly than the propagation corrections, and that the sources have a stronger dynamic patten than the propagations. In this fitting scheme, it is thus recommended that $\mu_{\epsilon}>\mu_{\delta}$.

For road traffic source category 1, the corrections for the vertical and scattered propagation paths are smaller than the corrections for the horizontal paths, which does not necessarily imply that the vertical and scattered paths are more accurately predicted, but could also be caused by the horizontal path dominating the overall propagation. For road traffic source categories 2 and 3, the corrections for the vertical and scattered propagation paths are more dominant. According to the source positions in the case study (as shown in Figure 3), multiple reflections and scattering would probably dominate the propagation for these categories, and thus are more likely to be adapted.

Figures 4 and 9 show that the source and propagation corrections for the industrial sources do not seem to converge over the period of several weeks that is considered here. According to Equations 12 and 14 , if the contribution of the non-traffic sources are mainly caused by one propagation path and the non-traffic sources are simplified by only a few point sources, the source and propagation corrections would be difficult to distinguish from each other. In this case, the model might not recognize whether the source or the propagation is responsible for the mismatch between the predicted and measured sound pressure level. More measurement positions closer to the sources that can separate the contribution strength of sources and different propagation paths are therefore necessary in this case. 

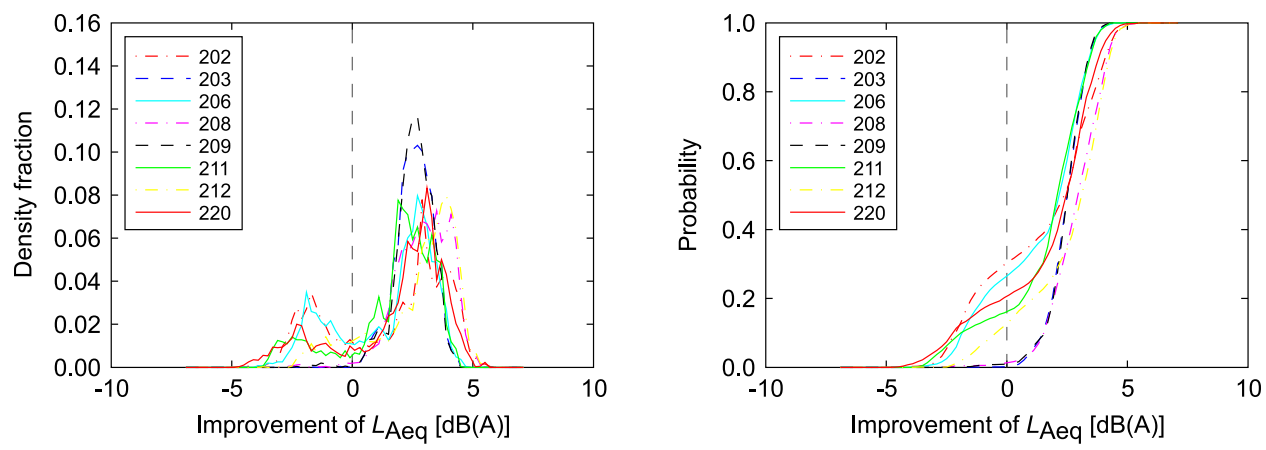

Figure 10: Distribution (left) and cumulative distribution (right) of the improvement on the predicted $L_{\text {Aeq. }}$.
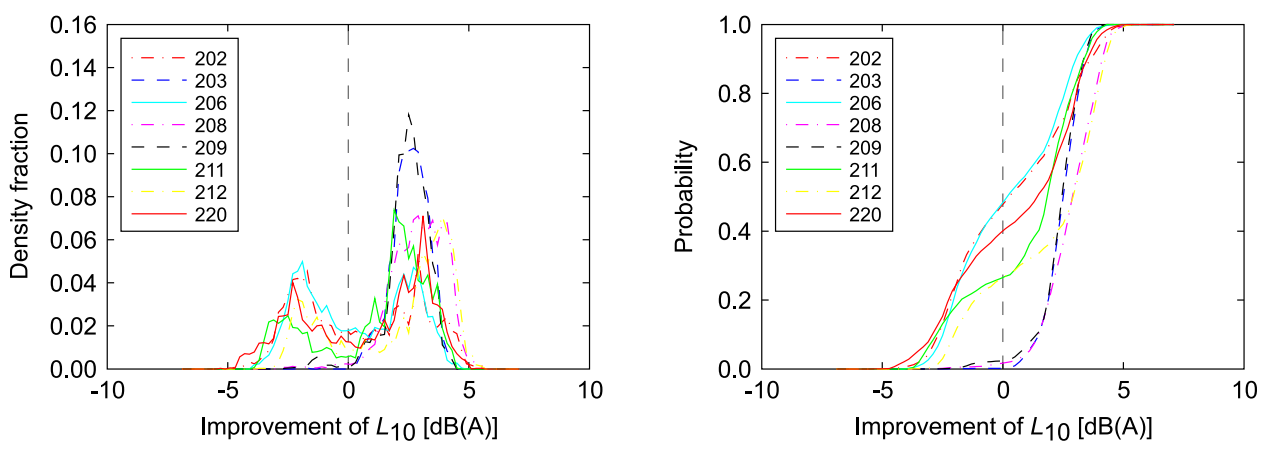

Figure 11: Distribution (left) and cumulative distribution (right) of the improvement on the predicted $L_{10}$.

\subsection{Validation}

Using the corrections adapted from the measurements, the predictions for $L_{A e q}$, both at the fitting locations and at the validation locations, are improved, as shown in Figures 10, 11 and 12. Negative values indicate that predictions become worse; positive values indicate that the prediction is improved after correcting. Figure 10 shows that more than $75 \%$ of the $L_{A e q, 15 \min }$ values at the validation positions matches measurements better than before correction. The improvement of $L_{\text {Aeq }}$ ranges typically from $0 \mathrm{~dB}$ to $5 \mathrm{~dB}$. For $L_{90}$, a similar improvement is achieved, as shown in Figure 12. However, the improvement of $L_{10}$ is not as good as for $L_{A e q}$ and $L_{90}$. The main reason is that the original predictions using the model mentioned in Section 2.1.3 were already quite accurate. Figure 13 shows the relation between the adapted and the original predictions of $L_{10}$. Clearly, the errors on most original predictions were already smaller than $5 \mathrm{~dB}$. Clearly, if the differences $L_{\text {Aeq }}-L_{\text {Aeq,meas }}$ and $L_{90}-L_{90, \text { meas }}$ push the corrections towards the opposite direction as compared to $L_{10}-L_{10, \text { meas }}$, the prediction of $L_{10}$ would become worse. Moreover, at moments and locations where the difference between measured and predicted $L_{10}$ is high, non-mapped sound sources may also contribute to the sound environment. However, the improved predictions still outweigh the worsened ones.

As an illustration of the proposed methodology, Figures 14 to 16 show snapshots of the $L_{A e q}$ noise 

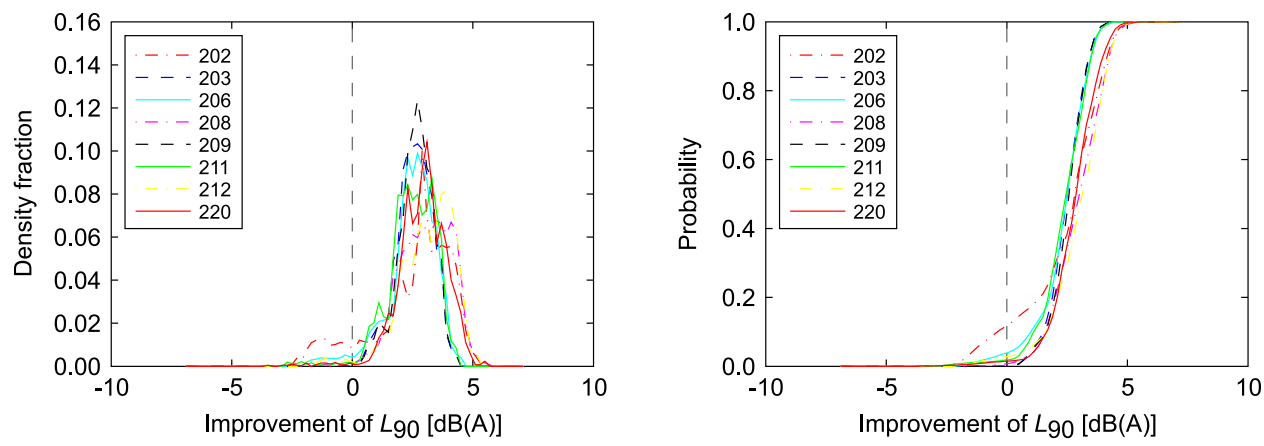

Figure 12: Distribution (left) and cumulative distribution (right) of the improvement on the predicted $L_{90}$.

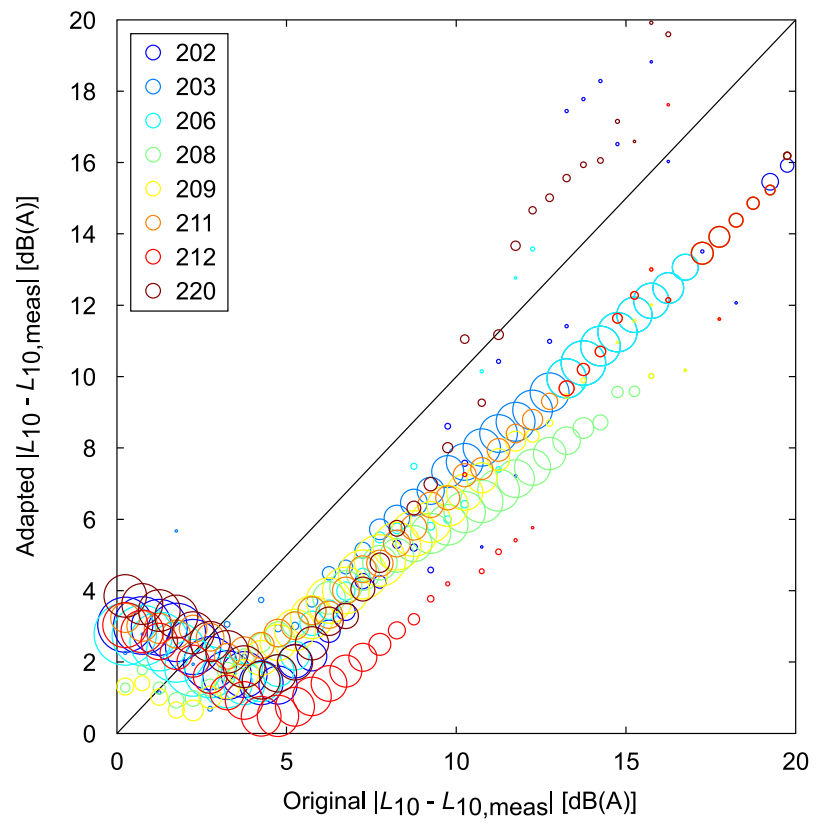

Figure 13: Original versus adapted predictions of $L_{10}$. The circle position indicates the error in the prediction; the circle radius indicates the number of predictions with this accuracy. 


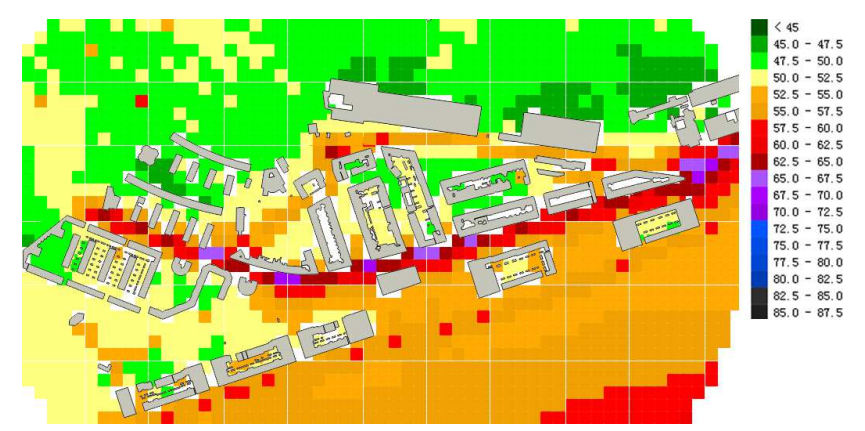

Figure 14: Snapshot of the noise map of $L_{\text {Aeq }}$ at 5:00 am.

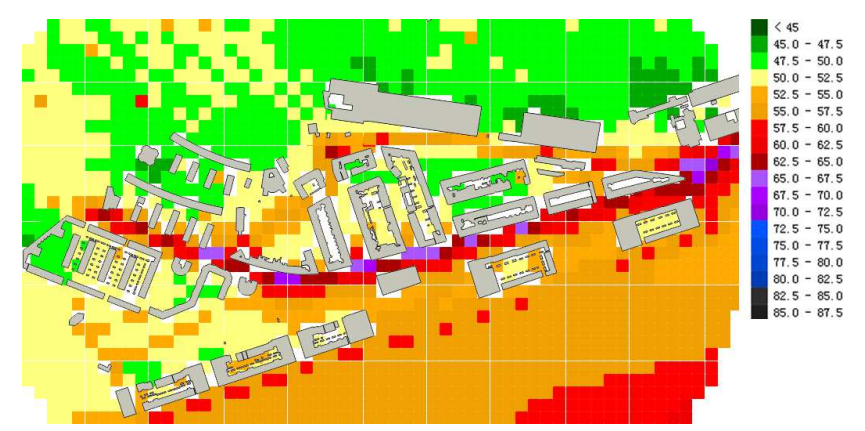

Figure 15: Snapshot of the noise map of $L_{A e q}$ at 6:00 am.

map on a particular day at 5:00 am, 6:00 am and 7:00 am. Whereas some dynamic changes between the noise maps at 5:00 am and at 6:00 am are captured by the model, the expected significant changes between 6:00 am and 7:00 am due to the morning rush hour inside the case study area can be observed easily. In this case study, 25498 sources and 3220 receivers were considered in total, and updating the map takes less than $0.5 \mathrm{~s}$ on an $\operatorname{Intel}(\mathrm{R}) \mathrm{Xeon}(\mathrm{R}) \mathrm{CPU}$ E5620 2.4 GHz. Given this update has to be performed only once every 15 minutes, the implemented approach shows good potential for scaling to much larger areas.

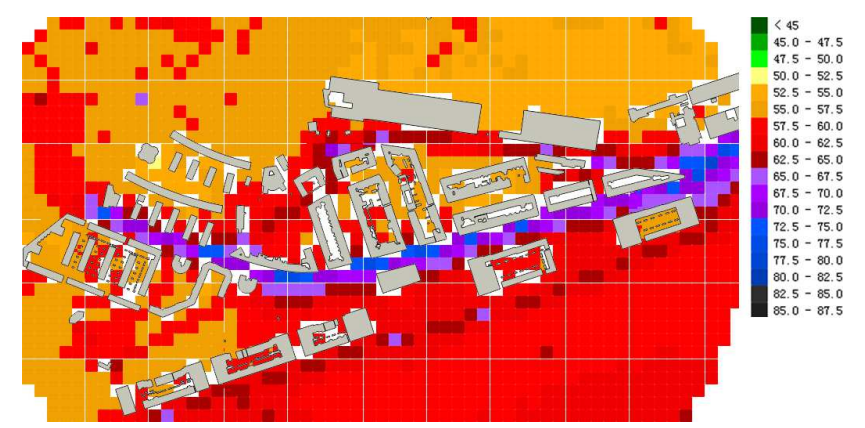

Figure 16: Snapshot of the noise map of $L_{\text {Aeq }}$ at 7:00 am. 


\section{Discussion and conclusion}

An improved noise mapping technique for interpolating between noise measurements in urban noise measurement networks is proposed. The underlying model distinguishes between horizontal (2D) propagation, vertical propagation, i.e. diffraction over buildings including multiple reflections in street canyons, and turbulence scattering. Calculation speed and accuracy are combined in the proces. In contrast to earlier work, the interpolation adapts not only source power but also propagation path attenuation. For this, the technique relies on optimal source grouping and on an LMS algorithm that slowly adapts the corrections needed, taking into account a regularization parameter that prevents over-fitting. Dynamic noise maps are obtained not only for $L_{A e q}$ but also for percentile noise levels, with $L_{10}$ and $L_{90}$ as an example. The analysis of the case study shows that this method could considerably increase correspondence between models and measurements for $L_{\text {Aeq }}$ and $L_{90}$. The main improvement range is between $0 \mathrm{~dB}$ and $5 \mathrm{~dB}$ with a peak at $3 \mathrm{~dB}$. The improvement on $L_{10}$ is not as good as for $L_{A e q}$ or $L_{90}$. The reason could be that the presented method to calculate $L_{10}$ is already quite accurate, but also that non-mapped sources could contribute. Although increasing the source power or decreasing the propagation attenuation can both increase the predicted noise level at receivers, this model can determine which of both should play a more important role due to the regularisation parameter. Additionally, this model can efficiently update the map every time step due to the use of pre-calculated partial maps.

In this study, the QSIDE model $[10,26]$ has been applied from the start to improve noise prediction in shielded areas, as compared to common practice in strategic noise mapping. Hence, the corrections are expected to be smaller than those that could be needed if a standard strategic noise map is used as a starting point. In the case study, the corrections $\epsilon$ and $\delta$ fall in the range from $0 \mathrm{~dB}$ to $5 \mathrm{~dB}$. The proposed model outperforms other approaches in its flexibility in the choice of measurement locations. Nevertheless, the uniqueness of the solution is improved if measurement stations are chosen in such a way that they characterize individual sources. Thus, in an ideal situation, one or more measurement stations should be close to each category of road traffic sources or industrial sources. As the measurements in the Katendrecht case study area were the result of a participatory process, the choice of measurement locations could not be optimized. The range of source and propagation corrections can be controlled by $\mu$ and $\beta$. For example, for smaller roads inside an urban area, traffic intensity data is typically not available. Therefore, the source correction should be amplified (with bigger $\mu$ or smaller $\beta$ ) and the propagation correction should be depressed (with smaller $\mu$ and bigger $\beta$ ). By doing so, the dynamic pattern of temporary sources or traffic can be captured. If the traffic intensity is known, such as for highways, the source correction should be depressed and the propagation correction should be amplified. In this study, a combination of different values for $\mu$ and $\beta$ was compared, and the best combination was used in the case study (see Section A.3).

The proposed approach for interpolating percentile noise levels is a first order approximation. Better performance could be obtained if the local dynamics in traffic noise is accounted for explicitly, taking into account for example vehicle platoons formed by the operation of traffic lights [23, 33]. However, this would have required a more dense network of measurement stations, which are currently being deployed in new case studies, and a refined approach for source corrections. In the Katendrecht case study, neither traffic lights nor traffic jams are present at close proximity to the dwellings, and hence these factors are not of importance and free traffic flow could be assumed. 


\section{Acknowledgements}

The measurement data used in this work was obtained through a pilot study commissioned by DCMR Milieudienst Rijnmond, Rotterdam, carried out within the framework of the Smart Sound Monitoring (SSM) research valorisation project, supported by the Industrial Research Fund (IOF) of Ghent University (IOF Advanced Grant 201).

\section{A Appendix}

\section{A.1 Adding different contributions to the overall level}

As introduced in Section 2, the total sound pressure level at receivers is obtained as a sum of terms originating from independent source categories and propagation paths. To calculate $L_{10}$ or $L_{90}$ of the sum of all contributions, the distribution of each of the individual contributions and their mutual correlations need to be known. As this information is generally not available, a simplified methodology is proposed. For each contribution, it is either assumed that the sound is constant or that its levels are normally distributed and independent from all other contributions. On the basis of a look-up table, traffic sound sources are considered to be constant if $\left|L_{10, m, n}-L_{e q, m, n}\right|<1 d B$ and $\left|L_{e q, m, n}-L_{90, m, n}\right|<1 d B$, where $m$ denotes the source category and $n$ denotes the propagation path category. For a constant sound, $L_{e q}=L_{10}=L_{90}$. When summing constant sounds, all percentile levels are simply equal to the energetic sum of all individual $L_{A e q}$ values.

Another common situation occurs when one contribution can be considered as constant, whereas the other one is fluctuating. In this situation, a good approximation for the percentile level of the total sound is obtained as:

$$
L_{x}=\left\{\begin{array}{lll}
L_{\text {eq, const }} & \text { if } & \mathrm{L}_{\text {eq }, \text { const }} \geq \mathrm{L}_{\mathrm{x}, \text { fluctuating }} \\
L_{x, \text { fluctuating }} & \text { if } & \mathrm{L}_{\text {eq, const }}<\mathrm{L}_{\mathrm{x}, \text { fluctuating }}
\end{array}\right.
$$

where $L_{\text {eq,const }}$ is the equivalent level of the constant source and $L_{x, \text { fluctuating }}$ is the percentile level of the fluctuating source.

If two or more contributions are fluctuating, and if it is assumed that the sound level at the receiver position satisfies a normal distribution, the relation between $L_{10}$ and $L_{e q}$ for a source $m$ and a propagation path $n$ can be calculated as:

$$
10^{0.1 L_{10, m, n}}-10^{0.1 L_{e q, m, n}}=1.28 \sigma_{m, n}
$$

where $\sigma$ is the standard deviation of the normal distribution. According to the properties of the normal distribution, the total $L_{10}$ and $L_{90}$ can then be written as:

$$
\begin{aligned}
& L_{10}=10 \log _{10}\left(\sum_{m} \sum_{n} 10^{0.1 L_{e q, m, n}}+1.28 \sqrt{\sum_{m} \sum_{n} \sigma_{m, n}^{2}}\right) \\
& L_{90}=10 \log _{10}\left(\sum_{m} \sum_{n} 10^{0.1 L_{e q, m, n}}-1.28 \sqrt{\sum_{m} \sum_{n} \sigma_{m, n}^{2}}\right)
\end{aligned}
$$


As a result, to calculate $L_{10}$ and $L_{90}$, the following procedure is followed: (i) group all contributions into two classes: "constant" and "fluctuating"; (ii) sum contributions within one class according to Equations 19 and 20; and (iii) calculate the overall $L_{10}$ and $L_{90}$ using Equation 17.

\section{A.2 Calculation of partial derivatives}

According to the formulation of $L_{10}$ and $L_{90}$, and the discussion in Section A.1, the derivative with respect to $\epsilon_{m}^{t-1}$ and $\delta_{m, n}^{t-1}$ equals zero for source category $m$ and propagation category $n$ for which the contribution is eliminated. If the dominating contribution to $L_{10}$ and $L_{90}$ belongs to the "constant" class, the derivative becomes the derivative of $L_{A e q}$, for which formulas have already been derived. If the dominating contribution belongs to the "fluctuating" class, according to Equation 19, the derivative $\frac{\partial}{\partial \epsilon_{m}^{t-1}} L_{10}$ becomes:

$$
\frac{\partial}{\partial \epsilon_{m}^{t-1}} L_{10}=\frac{10}{\ln 10} \frac{\frac{\partial}{\partial \epsilon_{m}^{t-1}} \sum_{m^{\prime}} \sum_{n} 10^{0.1 L_{e q, m^{\prime}, n}}+1.28 \frac{\partial}{\partial \epsilon_{m}^{t-1}} \sqrt{\sum_{m^{\prime}} \sum_{n} \sigma_{m^{\prime}, n}^{2}}}{\sum_{m^{\prime}} \sum_{n} 10^{0.1 L_{e q, m^{\prime}, n}}+1.28 \sqrt{\sum_{m^{\prime}} \sum_{n} \sigma_{m^{\prime}, n}^{2}}}
$$

Since $\sum_{m} \sum_{n} 10^{0.1 L_{e q, m, n}}$ is actually $\sum_{i}^{N_{s}} \sum_{j}^{N_{h}} 10^{0.1\left[L_{W, f, i}^{\prime}(t)-A_{f, i, j}^{\prime}(p, t)\right]}$ and accounting for Equation 10, one finds:

$$
\frac{\partial}{\partial \epsilon_{m}^{t-1}} \sum_{m} \sum_{n} 10^{0.1 L_{e q, m, n}}=\frac{\ln 10}{10} 10^{0.1 \epsilon_{m}^{t-1}} \sum_{n=1}^{N_{n}} 10^{-0.1 \delta_{m, n}^{t-1}} \sum_{j \in n} \sum_{i \in m} 10^{0.1\left[L_{W, f, i}(t)-A_{f, i, j}(p, t)\right]}
$$

The second part in the nominator of Equation 21 equals

$$
\frac{\partial}{\partial \epsilon_{m}^{t-1}} \sqrt{\sum_{m^{\prime}} \sum_{n} \sigma_{m^{\prime}, n}^{2}}=\frac{\sigma_{m, n}}{\sqrt{\sum_{m^{\prime}} \sum_{n} \sigma_{m^{\prime}, n}^{2}}}\left(\sum_{n^{\prime}} \frac{\partial}{\partial \epsilon_{m}^{t-1}} \sigma_{m, n^{\prime}}\right) .
$$

For the vertical path $(n=2)$ and the scattered path $(n=3)$, it can safely be assumed that the contribution belongs to the "constant" group, and thus these terms will never occur in the summation. Therefore, $\sum_{n} \frac{\partial}{\partial \epsilon_{m}^{t-1}} \sigma_{m, n} \approx \frac{\partial}{\partial \epsilon_{m}^{t-1}} \sigma_{m, 1}$. Substituting this approximation and Equation 18 into Equation 21 leads to:

$$
\begin{aligned}
\frac{\partial}{\partial \epsilon_{m}^{t-1}} \sigma_{m, 1} & =\frac{1}{1.28} \frac{\partial}{\partial \epsilon_{m}^{t-1}}\left[10^{0.1\left(L_{e q, m, 1}^{\prime}+\Delta_{10, m, 1}\right)}-10^{0.1 L_{e q, m, 1}^{\prime}}\right] \\
& =\frac{1}{1.28} \frac{\partial}{\partial \epsilon_{m}^{t-1}}\left\{10^{\epsilon_{m}^{t-1}-\delta_{m, 1}^{t-1}}\left[\sum_{i \in m} 10^{0.1\left(L_{W, f, i}-A_{f, i, 1}+\Delta_{10, m, 1}\right)}-\sum_{i \in m} 10^{0.1\left(L_{W, f, i}-A_{f, i, 1}\right)}\right]\right\} \\
& =\frac{\ln 10}{12.8} 10^{\epsilon_{m}^{t-1}-\delta_{m, 1}^{t-1}}\left(10^{0.1 \Delta_{10, m, 1}}-1\right) \sum_{i \in m} 10^{0.1\left(L_{W, f, i}-A_{f, i, 1}\right)}
\end{aligned}
$$

where, $L_{e q, m, 1}$ is the equivalent level of source category $m$ through propagation path 1 (the horizontal path) and $\Delta_{10, m, 1}$ is the level difference obtained from the tabulated results of typical traffic situations as explained before. While deriving Equation 24, it is assumed that $\sigma_{m, 1}$ is constant. This implies that the traffic intensity and speed are assumed constant and thus the difference between $L_{10}$ and $L_{e q}$ is constant. In other words, all inaccuracy is assumed to be related to the sound power emitted by each single vehicle. 
Similarly, $\frac{\partial}{\partial \epsilon_{m}^{t-1}} L_{90}$ can also be resolved as follows:

$$
\frac{\partial}{\partial \epsilon_{m}^{t-1}} L_{90}=\frac{10}{\ln 10} \frac{\frac{\partial}{\partial \epsilon_{m}^{t-1}} \sum_{m^{\prime}} \sum_{n} 10^{0.1 L_{e q, m^{\prime}, n}}-1.28 \frac{\sigma_{m, n}}{\sqrt{\sum_{m^{\prime}} \sum_{n} \sigma_{m^{\prime}, n}^{2}}} \frac{\partial}{\epsilon_{m}^{t-1}} \sigma_{m, 1}}{\sum_{m^{\prime}} \sum_{n} 10^{0.1 L_{e q, m^{\prime}, n}}-1.28 \sqrt{\sum_{m^{\prime}} \sum_{n} \sigma_{m^{\prime}, n}^{2}}}
$$

Finally, the terms $\frac{\partial}{\partial \delta_{m, n}^{t-1}} L_{10}$ and $\frac{\partial}{\partial \delta_{m, n}^{t-1}} L_{90}$ can be solved in a similar way as above:

$$
\begin{gathered}
\frac{\partial}{\partial \delta_{m, n}^{t-1}} L_{10}=\frac{10}{\ln 10} \frac{\frac{\partial}{\partial \delta_{m, n}^{t-1}} \sum_{m^{\prime}} \sum_{n^{\prime}} 10^{0.1 L_{e q, m^{\prime}, n^{\prime}}}+1.28 \frac{\sigma_{m, n}}{\sqrt{\sum_{m^{\prime}} \sum_{n^{\prime}} \sigma_{m^{\prime}, n^{\prime}}^{2}}} \frac{\partial}{\partial \delta_{m}^{t-1}} \sigma_{m, 1}}{\sum_{m^{\prime}} \sum_{n^{\prime}} 10^{0.1 L_{e q, m^{\prime}, n^{\prime}}+1.28 \sqrt{\sum_{m^{\prime}} \sum_{n^{\prime}} \sigma_{m^{\prime}, n^{\prime}}^{2}}}} \\
\frac{\partial}{\partial \delta_{m, n}^{t-1}} L_{90}=\frac{10}{\ln 10} \frac{\frac{\partial}{\partial \delta_{m, n}^{t-1}} \sum_{m^{\prime}} \sum_{n^{\prime}} 10^{0.1 L_{e q, m^{\prime}, n^{\prime}}}-1.28 \frac{\sigma_{m, n}}{\sqrt{\sum_{m^{\prime}} \sum_{n^{\prime}} \sigma_{m^{\prime}, n^{\prime}}^{2}}} \frac{\partial}{\partial \delta_{m}^{t-1}} \sigma_{m, 1}}{\sum_{m^{\prime}} \sum_{n^{\prime}} 10^{0.1 L_{e q, m^{\prime}, n^{\prime}}-1.28 \sqrt{\sum_{m^{\prime}} \sum_{n^{\prime}} \sigma_{m^{\prime}, n^{\prime}}^{2}}}}
\end{gathered}
$$

where

$$
\frac{\partial}{\partial \delta_{m, n}^{t-1}} \sum_{m^{\prime}} \sum_{n^{\prime}} 10^{0.1 L_{e q, m^{\prime}, n^{\prime}}}=-10^{0.1 \epsilon_{m}^{t-1}} 10^{-0.1 \delta_{m, n}^{t-1}} \sum_{j \in n} \sum_{i \in m} 10^{0.1\left[L_{W, f, i}(t)-A_{f, i, j}(p, t)\right]}
$$

and

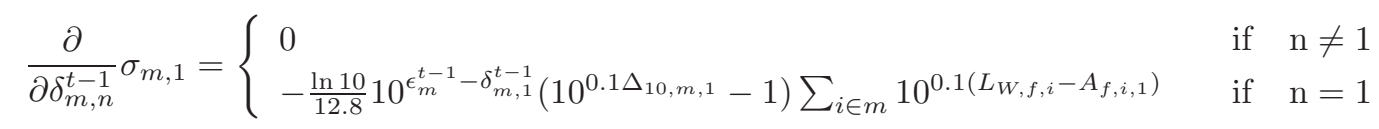

\section{A.3 Influence of propagation correction}

As mentioned in the previous sections, it is possible to update a noise map by only correcting source emissions. As this is the most common approach in map-based interpolation to date, the impact of also including corrections on propagation attenuation is investigated in more detail in this section. Figures 17 to 19 show the improvements by updating both source emission and propagation attenuation as compared to only updating the source emission. For this purpose, the proposed model is run twice on the same measurement period, once allowing $\epsilon$ and $\delta$ to adapt, and once keeping $\delta=0$. This results in an improvement with respect to measurements of $\Delta_{\epsilon, \delta}$ and $\Delta_{\epsilon}$ respectively. The distribution for all time intervals during 55 days is shown as a function of $\Delta_{\epsilon, \delta}-\Delta_{\epsilon}$ in Figure 17 . Positive values indicate that a better prediction was obtained by updating both source emission and propagation attenuation, whereas negative values indicate the opposite.

Figure 17 shows that correcting both source emission and propagation attenuation results in a better prediction of $L_{A e q}$ in around $80 \%$ of situations on average. Most of the improvements fall in the range from $0 \mathrm{~dB}$ to $1.2 \mathrm{~dB}$. For $L_{10}$, the propagation attenuation correction does not affect the prediction considerably, as shown in Figure 18. This is because sound peaks are typically caused by the closest road and through the horizontal propagation path, which is least likely to be incorrect. Therefore only modifying source emission can achieve similar improvement as compared to correcting both source emission and propagation attenuation. However, the propagation correction 

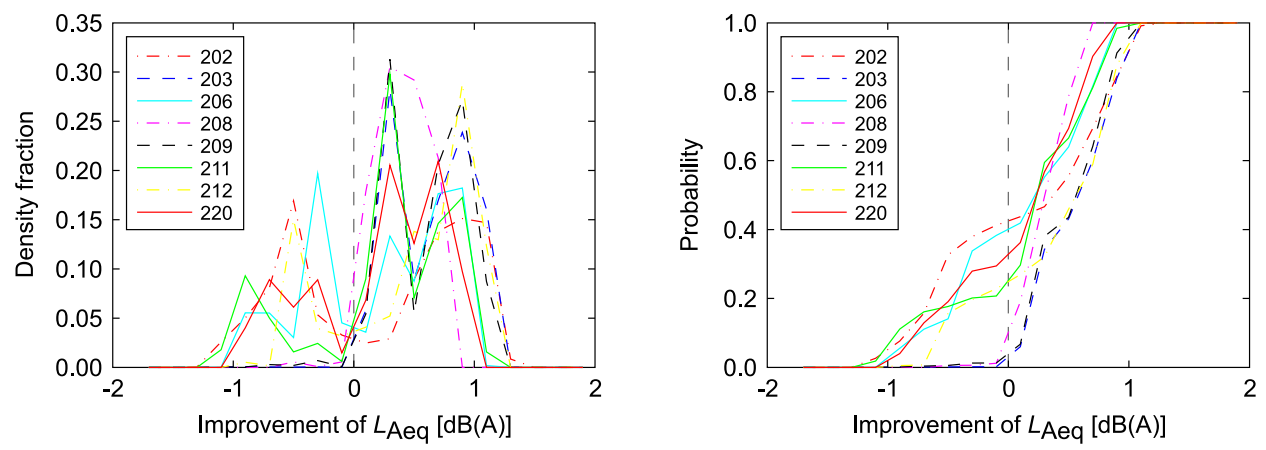

Figure 17: Comparison of $L_{A e q}$ between updating only sources and updating both sources and propagations; distribution (left) and cumulative distribution (right).
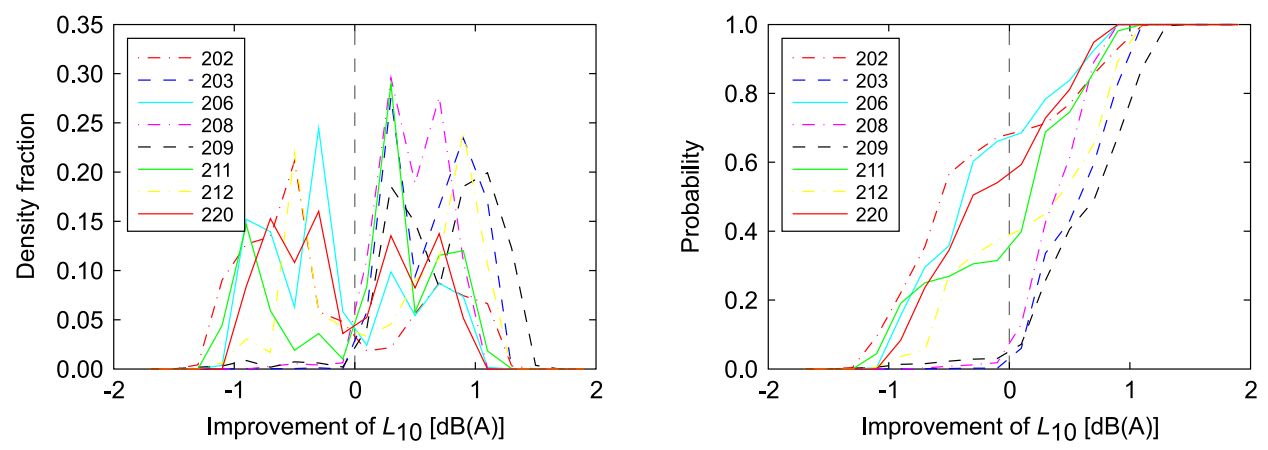

Figure 18: Comparison of $L_{10}$ between updating only sources and updating both sources and propagations; distribution (left) and cumulative distribution (right).

improves the $L_{90}$ prediction considerably. On average, more than $90 \%$ of situations result in a better prediction if both corrections are applied. The reason could be that $L_{90}$ is governed by the overall noise environment and not only by the traffic on the closest road, which is often a minor road in the residential area under study. The contribution of distant sources to $L_{90}$ plays a more important role and propagation corrections, such as diffraction over rooftops and turbulent scattering, may improve these.

\section{References}

[1] M. Á. Martín, Ana I. Tarrero, M. Machimbarrena, J. González, and V. González De Garibay. A methodology to study noise annoyance and to perform Action Plans follow up using as input an existing survey and noise map: Application to the city of Málaga (Spain). Applied Acoustics, 72(8):495-504, July 2011.

[2] P. H. Trombetta Zannin and D. Queiroz De SantAna. Noise mapping at different stages of a freeway redevelopment project A case study in Brazil. Applied Acoustics, 72(8):479-486, July 2011. 

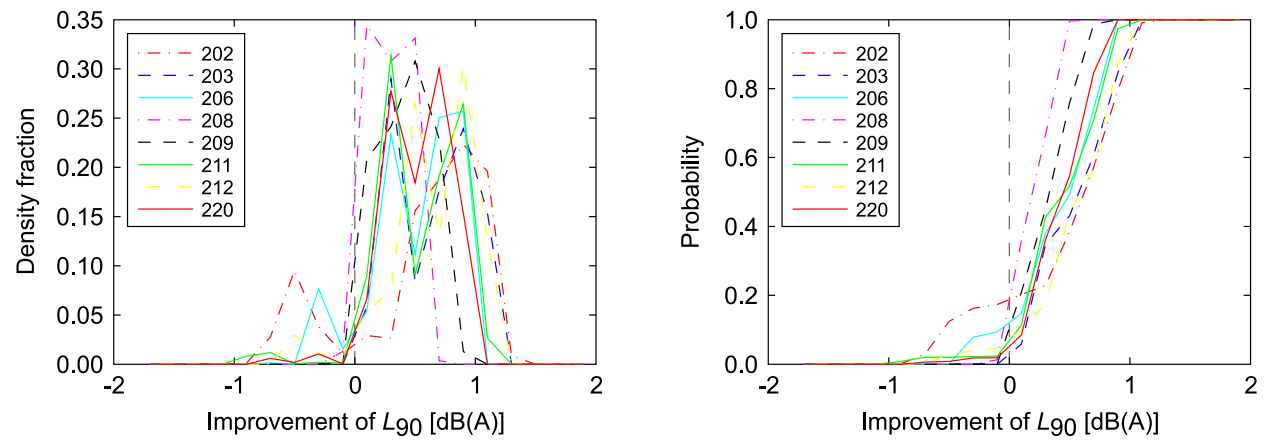

Figure 19: Comparison of $L_{90}$ between updating only sources and updating both sources and propagations; distribution (left) and cumulative distribution (right).

[3] J. H. Ko, S. I. Chang, and B. C. Lee. Noise impact assessment by utilizing noise map and GIS: A case study in the city of Chungju, Republic of Korea. Applied Acoustics, 72(8):544-550, July 2011.

[4] K. T. Tsai, M. D. Lin, and Y. H. Chen. Noise mapping in urban environments: A Taiwan study. Applied Acoustics, 70(7):964-972, 2009.

[5] B. Wang and J. Kang. Effects of urban morphology on the traffic noise distribution through noise mapping: A comparative study between UK and China. Applied Acoustics, 72(8):556-568, July 2011.

[6] P. Mioduszewski, J. A. Ejsmont, J. Grabowski, and D. Karpiski. Noise map validation by continuous noise monitoring. Applied Acoustics, 72(8):582-589, July 2011.

[7] J. M. Barrigón Morillas and C. Prieto Gajardo. Uncertainty evaluation of continuous noise sampling. Applied Acoustics, 75:27-36, 2014.

[8] M. Ögren and E. Salomons. Qside: http://www.qside.eu, October 2010.

[9] M. Schiff, M. Hornikx, and J. Forssén. Excess attenuation for sound propagation over an urban canyon. Applied Acoustics, 71(6):510-517, June 2010.

[10] W. Wei, D. Botteldooren, T. Van Renterghem, M. Hornikx, J. Forssén, E. Salomons, and M. Ögren. Urban background noise mapping: The general model. Acta Acustica united with Acustica, 100(6):1098-1111, 2014.

[11] T. Van Renterghem and D. Botteldooren. In-situ measurements of sound propagating over extensive green roofs. Building and Environment, 46(3):729-738, 2011.

[12] T. Van Renterghem and D. Botteldooren. The importance of roof shape for road traffic noise shielding in the urban environment. Journal of Sound and Vibration, 329(9):1422-1434, 2010.

[13] M. Arana, R. San Martin, M. L. San Martin, and E. Aramendia. Strategic noise map of a major road carried out with two environmental prediction software packages. Environmental Monitoring and Assessment, 163(1):503-513, 2010.

[14] M. Arana, R. San Martín, I. Nagore, and D. Pérez. What precision in the Digital Terrain Model is required for noise mapping? Applied Acoustics, 72(8):522-526, July 2011. 
[15] B. Griefahn. Environmental noise and sleep. Review-Need for further research. Applied Acoustics, 32(4):255-268, January 1991.

[16] G. Gereb. Real-time updating of noise maps by source-selective noise monitoring. Noise Control Engineering Journal, 61(2):228-239, March 2013.

[17] M. Szczodrak, J. Kotus, B. Kostek, and A. Czyzewski. Creating Dynamic Maps of Noise Threat Using PL-Grid Infrastructure. Archives of Acoustics, 38(2):235-242, January 2013.

[18] D. S. Cho, J. H. Kim, and D. Manvell. Noise mapping using measured noise and GPS data. Applied Acoustics, 68(9):1054-1061, September 2007.

[19] A. Can, L. Leclercq, J. Lelong, and D. Botteldooren. Traffic noise spectrum analysis: Dynamic modeling vs. experimental observations. Applied Acoustics, 71(8):764-770, August 2010.

[20] B. De Coensel, D. Botteldooren, F. Vanhove, and S. Logghe. Microsimulation based corrections on the road traffic noise emission near intersections. Acta Acustica united with Acustica, 93(2):241-252, March 2007.

[21] M. Xia-lin and C. Ming. Rendering of dynamic road traffic noise map based on Paramics. Procedia-Social and Behavioral Sciences, 96(Cictp):1460-1468, November 2013.

[22] A. Can, T. Van Renterghem, M. Rademaker, S. Dauwe, P. Thomas, B. De Baets, and D. Botteldooren. Sampling approaches to predict urban street noise levels using fixed and temporary microphones. Journal of Environmental Monitoring, 13(10):2710-9, October 2011.

[23] B. De Coensel, T. De Muer, I. Yperman, and D. Botteldooren. The influence of traffic flow dynamics on urban soundscapes. Applied Acoustics, 66(2):175-194, February 2005.

[24] B. De Coensel, F. Vanhove, S. Logghe, I. Wilmink, and D. Botteldooren. Noise emission corrections at intersections based on microscopic traffic simulation. In Proceedings of the 6th European Conference on Noise Control (Euronoise), Tampere, Finland, May 2006.

[25] B. De Coensel, D. Botteldooren, T. De Muer, B. Berglund, M. E. Nilsson, and P. Lercher. A model for the perception of environmental sound based on notice-events. Journal of the Acoustical Society of America, 126(2):656-665, August 2009.

[26] J. Forssén, M. Hornikx, T. Van Renterghem, W. Wei, D. Botteldooren, and M. Ögren. A model of sound scattering by atmospheric turbulence for use in noise mapping calculations. Acta Acustica united with Acustica, 100(5):810-815, September 2014.

[27] B. De Coensel and D. Botteldooren. Smart sound monitoring for sound event detection and characterization. In Proceedings of the 43rd International Congress and Exposition on Noise Control Engineering (Internoise 2014), Melbourne, Australia, 2014.

[28] G. F. Jenks. The data model concept in statistical mapping. International Yearbook of Cartography, 7(1):186-190, 1967.

[29] T. Van Renterghem, P. Thomas, F. Dominguez, S. Dauwe, A. Touhafi, B. Dhoedt, and D. Botteldooren. On the ability of consumer electronics microphones for environmental noise monitoring. Journal of Environmental Monitoring, 13(3):544-552, March 2011.

[30] B. De Greve, T. De Muer, and D. Botteldooren. Outdoor beam tracing over undulating terrain. In Proceedings of Forum Acusticum, Budapest, Hungary, August 2005. 
[31] G. Memoli, M. Paviotti, S. Kephalopoulos, and G. Licitra. Testing the acoustical corrections for reflections on a façade. Applied Acoustics, 69(6):479-495, June 2008.

[32] S. H. Tang and K. M. Li. The prediction of façade effects from a point source above an impedance ground. Journal of the Acoustical Society of America, 110(1):278-288, July 2001.

[33] A. Can, L. Leclercq, and J. Lelong. Dynamic estimation of urban traffic noise: Influence of traffic and noise source representations. Applied Acoustics, 69(10):858-867, October 2008. 